\title{
Development of the Fabrication of Graphene Liquid Cells for Transmission Electron Microscopy
}

\author{
Nathan Rosenmann ${ }^{1}$, Lopa Bhatt ${ }^{1}$, Jacob Jokisaari ${ }^{2}$, Mark Schlossman ${ }^{1}$ and Robert Klie ${ }^{1}$ \\ ${ }^{1}$ University of Illinois at Chicago, Chicago, Illinois, United States, ${ }^{2}$ Ted Pella, Redding, California, United \\ States
}

Pollution and water scarcity have put a great deal of pressure on the scientific community to find efficient and effective ways to clean polluted water. While current studies focus on developing new technologies to increase supplies of clean water, water purification could become more efficient if it was investigated and better understood at the molecular level. The initial stages of water purification focus on having the contaminant particles within water cluster together before being removed mechanically. The resulting water is then sent through membranes to be filtered. If particulates are not completely removed, the water sent through these membranes is not filtered properly and, therefore, is not safe for human consumption. Current treatments utilize aluminum $(\mathrm{Al})$ and iron $(\mathrm{Fe})$ salts as coagulants to form clusters of particulate contaminants and natural organic matter that is often charged. Yet much remains unknown about the behavior of charged nanoparticles in aqueous solution contained in nanoconfined space, primarily due to their small scale and the limitations of optical microscopes.

In order to overcome the limitation of the resolution of optical microscopy, we utilize transmission electron microscopy (TEM) to achieve high resolution imaging of nanoparticles in solution. As liquids cannot be place directly into the high vacuum environment of a TEM, studies have focused on developing membrane-based liquid cell stages which encapsulate liquid between thin silicon nitride membranes. While these developments have allowed for in-situ, electrochemical, and vapor-phase measurements to be possible, the thickness of window material limits the special resolution.

In this work, we will utilize gold $(\mathrm{Au})$ nanoparticles suspended in liquid to model the interactions of charged nano-particles in water. The goal for this study is to quantify the aggregation of Au particles that takes place in the early stages of water purification by utilizing in-situ scanning transmission electron microscopy (STEM). [1,2] We have developed a fabrication method for graphene-based liquid cells (GLCs) that allows for higher special resolution than their silicon nitride counterparts while also minimizing surface contamination that could disturb the nanoparticles within them (Figure 1). [3, 4] These GLCs contain small quantities of gold nanoparticles in solution sandwiched between two single layer (or 3-5 layer) graphene sheets (Figure 1). Although it is only a single atomic-layer thick, the graphene prevents water from escaping into the vacuum chamber of the STEM. In addition, the low electron scattering of the graphene allows for the imaging of nanoparticles in the encapsulated water by STEM without much interference. [5]

The process of fabricating GLCs is shown schematically below (Figure 2): The Cu support from the CVDgrown graphene samples are etched for 24 hours. Following the etching, the graphene is transferred to ultrapure water for an additional 24 hours. A plasma cleaned (hydrophilic) $\mathrm{Cu}$-mesh TEM grid is then dipped onto the surface of the floating graphene and set to dry under a heat lamp. The liquid sample is then deposited on the heated graphene before using a humidifier to deposit small droplets of water over the surface of the graphene-coated grid. Following this step, the sample is dipped immediately onto a second graphene window layer to fully encapsulate the solution.

The implementation of fabrication steps to increase the volume of water encapsulated within the cell while optimizing spatial resolution will be discussed. Additionally, we will analyze the role that electrostatic 
interactions play in the aggregation and movement of gold nanoparticles within GLCs using STEM imaging. The goal is to utilize this optimized approach for creating GLCs to better reveal the interactions of charged nanoparticles and their role in water purification. [6]
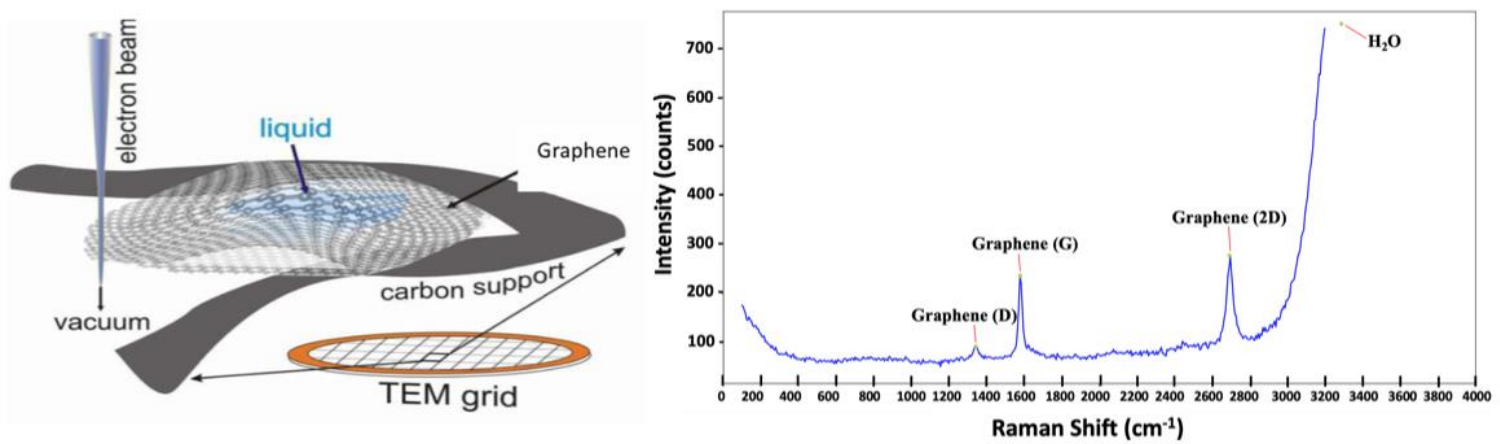

Figure 1. Diagram depicting a liquid cell formed within a grid hole of a TEM grid (left) [5]; Raman Spectrum of floating etched graphene film utilized for GLCs [3].

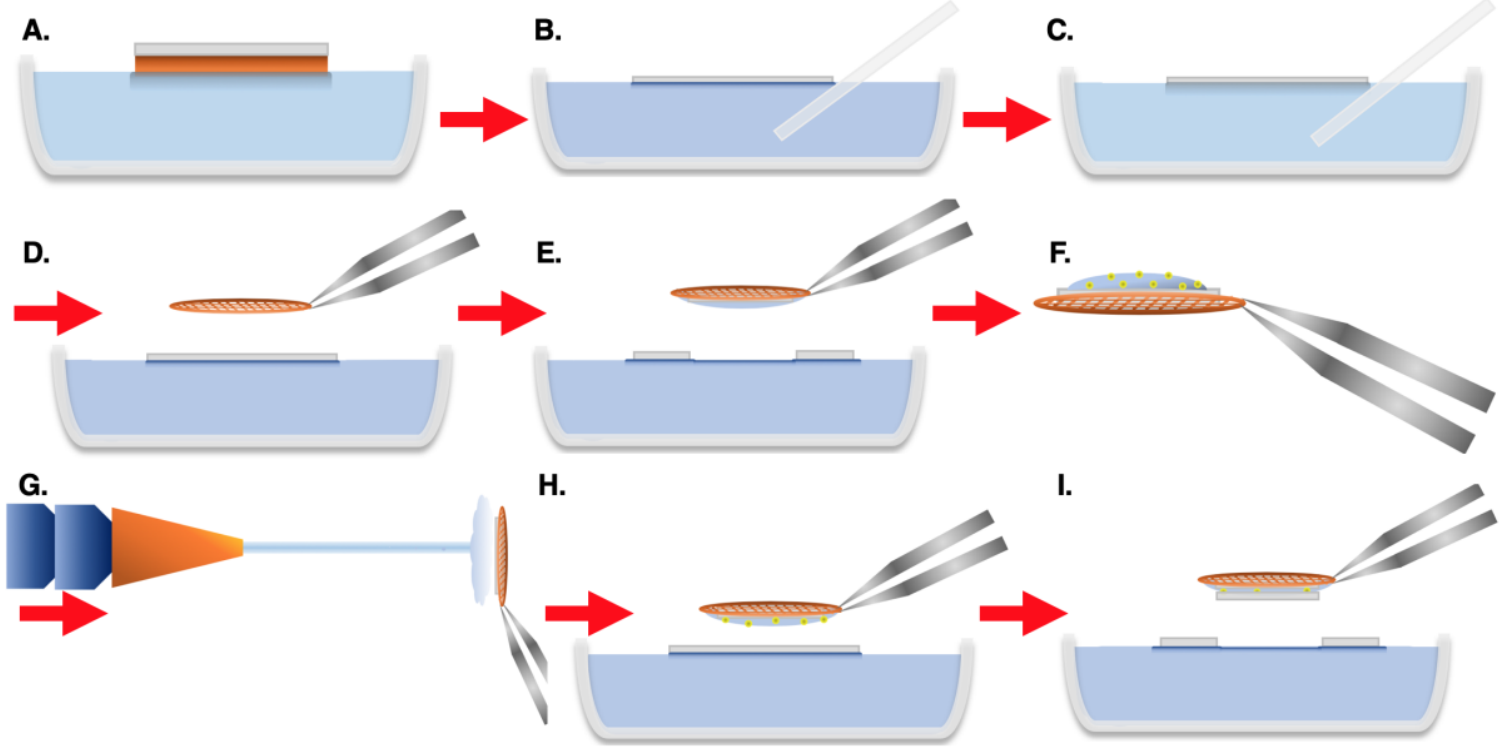

Figure 2. Schematic describing the method developed to fabricate GLCs.

\section{References}

[1] C. Wang et al., Adv. Mat., 26(21) (2014)

[2] H. Cho et al., Nano Lett., 17, 414-420. (2017)

[3] N. Rosenmann et al., Microscopy and Microanalysis, 25, 2096-2097, (2019)

[4] L. Bhatt et al., Microscopy and Microanalysis, 25, 2144-2145, (2019)

[5] J. Jokisaari et al., Microscopy and Microanalysis, 23, 878-879 (2017)

[6] This work was in part supported by the National Science Foundation (EFMA-1542864 (EFRI)) and by the Chancellor's Undergraduate Research Award 